УДК 65.01

АДАПТИВНЫЕ ОРГАНИЗАЦИОННЫЕ СИСТЕМЫ И ИХ МОДЕЛИРОВАНИЕ

1Черников Б.В., ${ }^{2}$ Антончиков С.Н.

${ }^{1}$ ООО «Газпром ВНИИГАЗ», Российский экономический университет им. Г.В. Плеханова, Москва, e-mail: bor-cher@yandex.ru;

${ }^{2}$ МГТУ им. Н.Э. Баумана, Москва, е-mail: sergeyantonchikov@gmail.com

\begin{abstract}
В работе сформирован общий подход к моделированию адаптивных организационных систем. В основу подхода положена возможность представления организационной системы в виде метакортежа, состоящего из частных кортежей частных моделей, характеризующих определенные уровни ее организации, одновременно обладающих заданным свойством. В качестве инструмента обеспечения адаптивности рассматривается соответствующее управление. Поэтому создание модели адаптивной организационной системы предполагает формирование метакортежа, включающего частные кортежи частных моделей адаптивного управления на каждом из уровней организации. Для выбора элементов метакортежа - частных кортежей, могут использоваться различные системы организационной классификации. Одной из ключевых особенностей адаптивного управления организацией выступает неопределенность. Разрешение неопределенности является основной задачей адаптивных организационных систем, что предполагает внедрение соответствующего механизма в каждую из частных моделей адаптивного управления организации.
\end{abstract}

Ключевые слова: адаптивность, кортеж, метакортеж, модель, моделирование, неопределенность, организационная система, организация, управление

\title{
ADAPTIVE ORGANIZATIONAL SYSTEMS AND THEIR MODELING \\ ${ }^{1}$ Chernikov B.V., ${ }^{2}$ Antonchikov S.N. \\ ${ }^{1} L L C$ «Gazprom VNIIGAZ», Plekhanov Russian University of Economics, Moscow, e-mail: bor-cher@yandex.ru; ²Bauman Moscow State Technical University, Moscow, e-mail: sergeyantonchikov@gmail.com
}

General approaches to the modeling of adaptive organizational systems are formed in this paper. The approach is based on the possibility of the organizational system representing with a form of metatuple, which consists of particular tuples of particular models. As an instrument for ensuring adaptability, appropriate management is considered. Therefore, the creation of the model of an adaptive organizational system presupposes the formation of a metatulpe that includes particular tuples of particular models of adaptive management at each level of the organization. Various systems of organizational classification can be used to select the levels of organization of metatuple elements - particular tuples. One of the key features of adaptive management of the organization is uncertainty. Resolution of uncertainty is the main task of adaptive organizational systems. This implies the introduction of an appropriate mechanism in each of the particular models of the organization adaptive management.

Keywords: adaptivity, tuple, metatuple, model, modeling, uncertainty, organizational system, organization, management

Окружающий мир бесконечен в своем разнообразии. И это - объективное препятствие в его познании и изменении в интересах человека, т.е. в практической деятельности. На первый взгляд кажется очевидным, что если не существует двух абсолютно одинаковых предметов или явлений материального мира, то для решения очередной проблемы необходим абсолютно новый инструмент или средство. Тем не менее на протяжении всего периода эволюции человек создавал объекты, обладавшие определенными стабильными свойствами, и с их помощью решал возникающие перед ним задачи. Это были копии предметов, создаваемые по образцу, ранее обнаружившему полезные качества. Использование копии в ситуации, подобной произошедшей в прошлом, давало необходимый результат (хотя бы частичный), что и определяло ее востребованность. По сути это и были первые модели. Как было установлено в дальнейшем, в процессе развития научных исследовательских методов, моделирование является не только эффективным инструментом практической деятельности, но и средством познания окружающей действительности. Таким образом, модели обладают как прагматической, так и познавательной ценностью.

Широкая применимость моделей объясняется наличием у них ряда свойств. Среди них: упрощенность, приближенность, адекватность. В самом деле, несмотря на большое количество характеристик, которыми обладает оригинал, при решении определенной задачи интерес для человека представляют лишь некоторые свойства объекта. Их мы и воссоздаем в модели, игнорируя или упрощая все остальные качества. Приближенность модели позволяет создавать объект, повторяющий оригинал лишь с определенной степенью точности. Это значительно облегчает создание необходимого нам предмета, т.к. воспроизводство стопроцентной копии некоторых 
сложных объектов представляло бы невыполнимую задачу. Адекватность модели заключается в ее достаточности для достижения определенных целей, которые мы преследуем при воспроизведении реального объекта. Как указывается в работе [1]: «...Ф.И. Перегудов и Ф.П. Тарасенко отмечают... весьма важную особенность моделей, обеспечивающую их успешное применение на практике, - ингерентность модели, т.е. согласованность, совместимость модели с окружающей средой».

Отмеченные свойства моделей делают их незаменимыми при исследовании таких объектов, как организационные системы. В целях уточнения объекта исследования введем определение организационной системы. Для этого воспользуемся формулировкой, приведенной в работе [2], где указано, что организационные системы представляют собой «...объединение людей, совместно реализующих некоторую программу или цель и действующих на основе определенных процедур и правил».

Очевидно, что создание в качестве модели реальной организации как в исследовательских целях, так и для решения практических задач (при отсутствии верифицированных данных о применимости и эффективности модели), нецелесообразно. Это связано не только со сложностью самой решаемой задачи, но также и по экономическим соображениям. Кроме того, этому могут препятствовать законодательные и иные институциональные ограничения, в том числе морально-этического характера. Таким образом, наиболее эффективным инструментом исследования и выработки решений, связанных с созданием, управлением и развитием организационных систем, выступает их моделирование.

\section{Актуальность и цель исследования}

В последнее время запрос общества на повышение гибкости различных его элементов, в том числе организационных систем, звучит все более отчетливо. Выражаясь научной терминологией, речь идет о таком свойстве систем, как адаптивность.

Общественный интерес к развитию адаптивных качеств элементов социума не случаен. Причина его кроется в особенностях текущего этапа развития человечества. А именно, в усложнении устройства современного общества и в ускорении его развития под влиянием научно-технического прогресса. Обе тенденции являются источником все большего количества перемен, оказывающих непосредственное влияние на всех участников социальноэкономической системы. Возрастающие по силе и скорости изменения среды требуют адекватной реакции ее элементов. Иначе говоря, участникам социума приходится приспосабливаться к более частым, сложным и оказывающим существенное влияние на их существование переменам. Учитывая тот факт, что подобные события перестают быть единичными на горизонте жизненного цикла общественных систем, необходимо говорить об их готовности и способности к непрерывному приспособлению к внешним и внутренним возмущениям, т.е. к формированию некоего особого свойства системы. Масштаб и сила отдельных изменений среды способны вызвать не просто ухудшение условий, но даже могут в целом поставить вопрос прекращения существования некоторых элементов социума. Это требует развития таких адаптивных свойств участников общества, которые обеспечат их выживание в условиях существенных изменений факторов среды.

Таким образом, адаптивность общественных систем становится важным качеством, обеспечивающим их эффективное и долгосрочное существование на данном этапе развития социально-экономической формации.

Учитывая частое использование в литературе близких по значению и даже похожих терминов, таких как «адаптация», «адаптируемость», необходимо ввести более четкое понятие адаптивности. Для этого приведем соответствующие трактовки и определения. Развернутый терминологический анализ понятий, связанных со свойствами адаптивности систем, проведен в работе [3].

1. «Адаптивность - свойство системы приспосабливаться к изменяющимся условиям среды (помехам, возмущениям), которое нацелено на оптимизацию ее функционирования и в конечном счете - на сохранение системы как упорядоченной совокупности взаимодействующих элементов» [3].

2. «Предлагается под адаптацией понимать соответствующий процесс, связанный с реакцией системы на изменение внешних либо внутренних факторов. Адаптивность будет рассматриваться как некоторое свойство системы, направленное на восстановление равновесия, нарушенного изменением внешних либо внутренних факторов среды, т.е. на сохранение системы» [4].

В связи с тем, что адаптивность рассматривается как свойство организационных систем, использование методов моделирования представляется еще более обоснованным. Действительно, исследование свойств адаптивности различных моделей, представляющих одну и ту же организационную 
систему, позволит выявить их особенности и расширит понимание адаптивности организационных систем в целом.

Таким образом, целью данной работы является исследование адаптивности организационных систем и выработка основ методологии создания модели адаптивной организационной системы.

\section{Общий подход при построении модели адаптивной организационной системы}

В XX веке наука обогатилась новой мощной концепцией, получившей название системный подход, который затем трансформировался в эффективную методологическую платформу системного анализа. Это позволило совершить переворот в научных исследованиях благодаря формированию новых аналитических методов и технологий системного анализа, в том числе моделирования.

Однако, прежде чем заняться созданием моделей и затем использовать их для изучения свойств реальных объектов, необходимо разобраться, что они собой представляют. Для этого рассмотрим этимологию понятия «модель» и ее сущность. Согласно [5] слово «модель» произошло от латинского «modulus», что означает мера, образец, норма. Можно сделать вывод, что на латыни слово модель в большей степени употреблялось в его прагматическом значении. Модель рассматривалась в качестве образца для преобразования предметов окружающего мира с целью придания им необходимых свойств, т.е. для практических нужд. В работе [1] приводятся следующие определения модели.

1. «Под моделью данной системы понимается любая другая система, обладающая той же формальной структурой при условии, что:

- между системными характеристиками (функцией, структурой, элементами) модели и оригинала существует соответствие;

- модель более доступна для оперирования имеющимися средствами, чем оригинал».

2. «Моделью называется некий объект-заместитель, который в определенных условиях может заменять объект-оригинал, воспроизводя интересующие субъекта свойства и характеристики оригинала, и имеющий существенные преимущества перед оригиналом (наглядность, обозримость, доступность и т.д.)».

Следует отметить одну важную характеристику модели, зафиксированную во втором определении, которая представляет интерес в рамках данной работы. Существенно, что из всего многообразия свойств объекта-оригинала модель позволяет отражать лишь те из них, которые важны в рамках конкретного исследования. Это и обеспечивает преимущества модели перед оригиналом, в том числе такие, как наглядность, доступность и т.д. Кроме того, как указывает А.Н. Туликов в работе [6], «...модель является не просто образом - заменителем оригинала, не вообще каким-то отображением, а отображением целевым». Автор говорит о том, что в зависимости от профессионального запроса (цели деятельности) в одном и том же предмете каждый специалист может видеть средство для удовлетворения его уникальной профессиональной потребности.

Согласно [5] «...под моделированием следует понимать процесс адекватного отображения наиболее существенных сторон исследуемого объекта или явления с точностью, которая необходима для практических нужд».

Используя принципы системного подхода, опираясь на сущность и свойства моделей, попробуем выработать некоторый подход, применимый для настоящего исследования, т.е. для построения модели адаптивной организационной системы.

Предположим, что некоторый объекторигинал выступает носителем определенного свойства, которое и представляет интерес для исследования. Моделирование позволяет создавать некоторое (теоретически неограниченное) множество моделей одного и того же реального объекта, каждая из которых является отображением объекта-оригинала в определенном аспекте (например, на определенном уровне его организации). В свою очередь, некоторые из полученных моделей могут проявлять изучаемое свойство на своих уровнях организации объекта и поэтому будут являться предметом для дальнейшего исследования. Следовательно, изучение моделей, представляющих разные стороны объекта-оригинала и обладающих искомым свойством, позволит получить лучшее представление о механизмах реализации свойства оригинала в целом.

Принимая во внимание сложность того или иного исследуемого объекта, очевидно, что для представления уровней его организации может потребоваться формирование кортежа частных моделей:

$$
K=\left\{M_{1}^{1}, \ldots, M_{r}^{d}, \ldots, M_{R}^{D}\right\},
$$

где $M_{r}$ - частные модели объекта-оригинала, обладающие заданным свойством; $r=1$, $2, \ldots, R$ - номер-идентификатор частной модели; $d=1,2, \ldots, D$ - номер, определяющий местоположение элемента в кортеже. 
В этой связи подход может рассматриваться в качестве метода исследования некоторого свойства реального объекта, одновременно обнаруживаемого у моделей этого объекта, а также метода моделирования объекта, обладающего требуемым свойством.

Учитывая, что сложные и большие реальные системы, как правило, имеют более одного уровня организации, становится понятным, что для их представления требуется формирование метакортежа, т.е. кортежа, элементами которого будут выступать частные кортежи, состоящие из частных моделей, фиксирующих определенные стороны объекта оригинала, обладающие заданным свойством. Такой метод позволяет организовать дальнейшее исследование данного свойства благодаря каждой из полученных моделей.

Поскольку организационные системы относятся к классу больших и сложных систем, данный подход может быть применен и для изучения свойства их адаптивности. Метод может использоваться в качестве инструмента создания моделей организационных систем, обладающих свойством адаптивности. По своей природе это будут модели, представляющие собой метакортежи, включающие в себя упорядоченные множества частных кортежей частных моделей, обладающих заданным свойством.

$$
M K=\left\{K_{1}^{1}, \ldots, K_{h}^{g}, \ldots, K_{H}^{G}\right\},
$$

где $K_{h}=\left\{M_{1}{ }^{1}, \ldots, M_{r}^{d}, \ldots, M_{R}{ }^{D}\right\}$ - частные кортежи частных моделей $M_{r}^{R}$ объекта-оригинала, обладающих заданным свойством; $r=1,2, \ldots, R$ - номер-идентификатор частной модели; $d=1,2, \ldots, D$ - номер, определяющий местоположение элемента частного кортежа; $h=1,2, \ldots, H$ - номеридентификатор частного кортежа (количество и состав элементов каждого частного кортежа могут быть разными); $g=1,2, \ldots$, $G$ - номер, определяющий местоположение элемента метакортежа.

Графическое изображение модели организационной системы в форме метакортежа представлено на рис. 1 , где $M_{r}$ - частные модели; $K_{h}$ - частные кортежи частных моделей $M_{r} ; r=1,2, \ldots, R$ - номер-идентификатор частной модели; $d=1,2, \ldots, D$ - номер, определяющий местоположение элемента частного кортежа; $h=1,2, \ldots, H$ - номеридентификатор частного кортежа; $g=1$, $2, \ldots, G$ - номер, определяющий местоположение элемента метакортежа.

В свою очередь, наличие необходимого свойства у каждой из частных моделей в метакортеже будет определять проявление заданного свойства на уровне организации реального объекта (частного кортежа) и, соответственно, на уровне объекта в целом. В таком случае новая модель и будет представлять собой объект, обладающий требуемым свойством.

Согласно принятому определению, адаптивность рассматривается как свойство системы приспосабливаться к изменениям условий среды. Иными словами, это способность системы изменяться под воздействием внешних или внутренних возмущений. Одним из инструментов, способных обеспечивать изменение системы, является управление. Таким образом, адаптивность системы может обеспечиваться соответствующим управлением. Поэтому для построения моделей адаптивности системы требуется в том числе формирование моделей управления изменениями системы. Модель адаптивной организационной системы будет представлять собой метакортеж, состоящий из упорядоченного множества частных кортежей частных моделей управления адаптивностью организационной системы.

Таким образом, в качестве подхода к построению модели адаптивной организационной системы может рассматриваться формирование метакортежа, состоящего из частных кортежей частных моделей управления адаптивностью такой системы на различных уровнях ее организации. В связи с тем, что реализация свойства адаптивности организационной системы может быть обеспечена соответствующим управлением, создание адаптивной организационной системы предполагает формирование частных моделей адаптивного управления на каждом уровне системы. Целью управления является управление изменениями организационной системы под воздействием внешних или внутренних возмущений.

Целесообразно проверить применение положений данного подхода при моделировании адаптивной организационной системы.

\section{Адаптивность организационных систем на уровне базовых моделей}

Одним из элементов методологии системного анализа являются базовые модели систем. Применим некоторые из них в интересах исследования адаптивности организационных систем. Для этого воспользуемся моделями состава и структуры системы. Тем более что, как указывают авторы работы [5], «...эти виды моделей широко используются для формирования моделей организаций». 


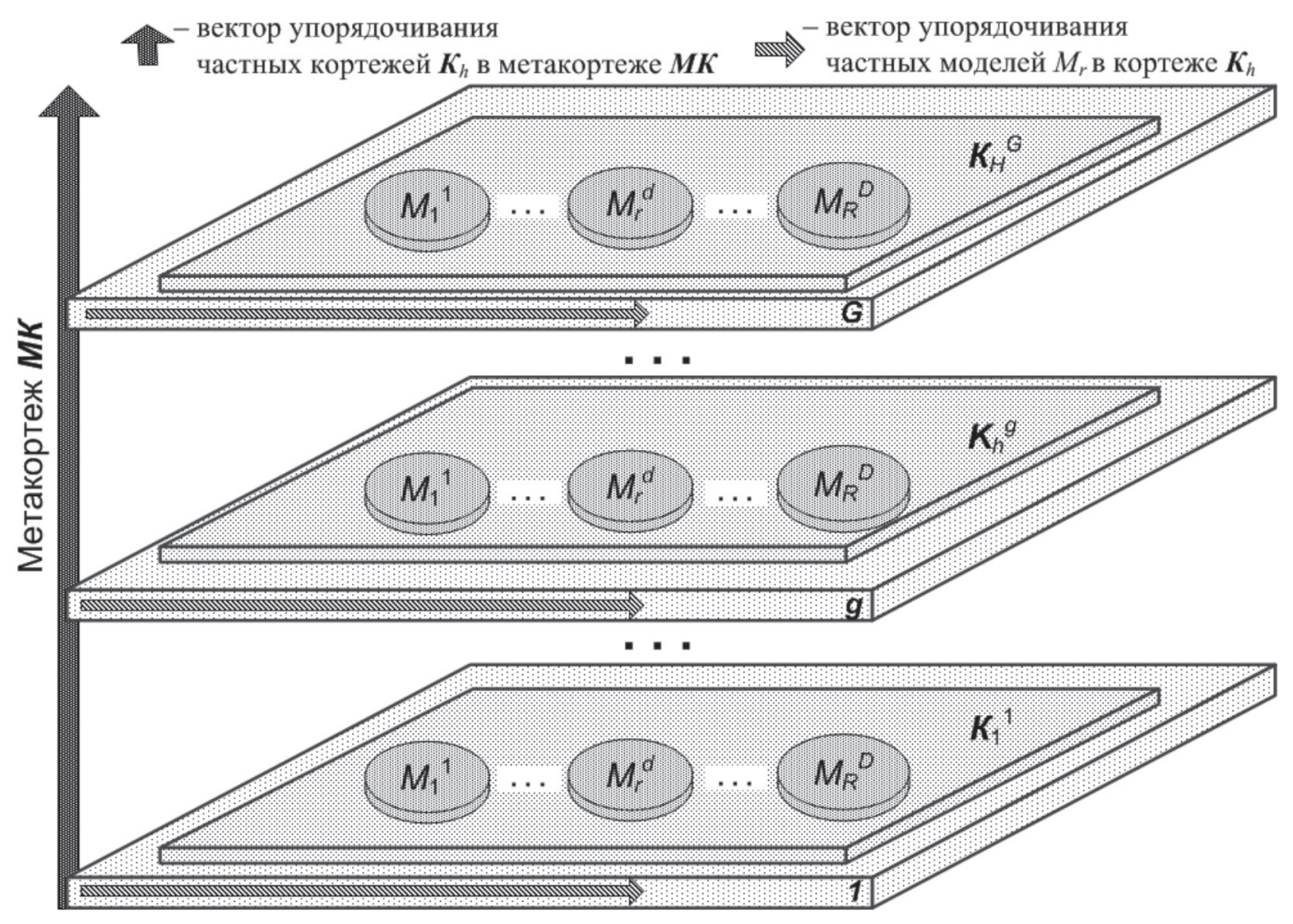

Рис. 1. Модель организационной системы в форме метакортежа МК

Модель состава системь. Прежде чем рассматривать данную модель, коротко вспомним основные свойства системы. Это позволит лучше представить, благодаря чему системы выделяются из окружающей среды, т.е. уточнить, как они формируются и, соответственно, из чего состоят.

Одними из основных свойств, которыми обладает система, выступают свойства целостности и интегративности. Они проявляются через такие характеристики системы, как обособленность (отграниченность) от окружающей среды, единство компонентов и целей системы, эмерджентность, наличие системообразующих и системосохраняющих факторов. В то же время системам присущи и аддитивные свойства, обусловленные тем, что система является совокупностью подсистем и элементов. Следует отметить, что количество составляющих систему элементов и частей различается не только в разрезе классов систем, но может существенно варьироваться и в границах одного класса. Это проявляется и в категории исследуемых организационных систем. Кроме того, элементный состав системы может отличаться значительной неоднородностью. По степени однородности состава системы принято делить на гомогенные, гетерогенные и сме- шанного типа. В свою очередь отдельные элементы системы могут обладать различными качествами, в том числе уникальными, отсутствующими у других элементов.

Для детального представления и изучения внутреннего состава систем формируются модели состава. Модели состава могут быть представлены в различном виде. Наиболее часто модели состава представляют в виде перечней, в табличном формате, а также в виде графических моделей. Распространенным примером модели состава организации является ее штатное расписание. Модель состава системы в графическом виде представлена на рис. 2.

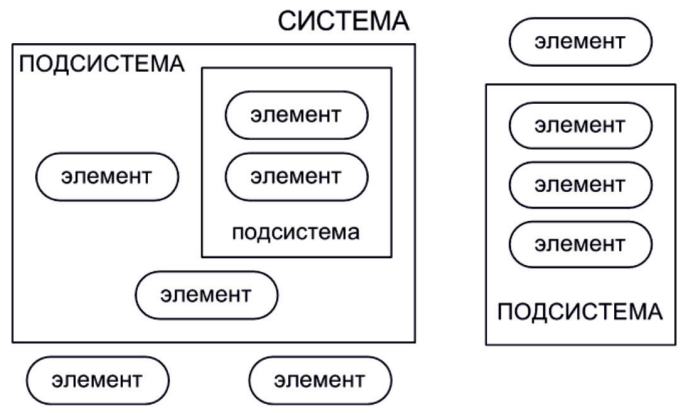

Рис. 2. Модель состава системь 
Формирование моделей состава системы позволяет подойти к решению задачи управления составом. Необходимо отметить, что управление составом организационных систем не является новой задачей и уже имеет наработанные подходы в рамках различных теорий. Так, с позиций научной организации труда, обычно используемой на практике, при определении потребности предприятия в работниках соответствующей категории учитывают плановые объемы производства, трудоемкость продукции, балансы рабочего времени среднесписочного работника, нормы обслуживания, графики сменности и т.д. Метод позволяет провести расчет плановой потребности в работниках для выполнения производственной программы. Существуют и иные способы решения данной задачи. Д.А. Новиков в работе [7] при рассмотрении прочих известных подходов управления численностью отметил следующее. Модели определения оптимального числа работников исследовались, например, в теории контрактов при ограничениях согласованности стимулирования и резервной заработной платы. Оптимальное количество работников в рамках экономики труда определяется исходя из равенства производимого ими предельного продукта (предельной производительности) и предельных затрат на привлечение работников. Согласно подходам экономики организации оптимальная численность работников может быть выведена на основе сравнения транзакционных издержек участников рынка и организационных издержек компании, возникающих в том числе при найме и удержании персонала. Задачи формирования состава организации рассматривались также в теории активных систем, в теории графов. Для решения таких задач могут применяться конкурсные и аукционные механизмы.

Следует отметить, что управление численностью - не единственный вопрос, требующий решения при управлении составом организации. В работе [7] Д.А. Новиков приводит следующую классификацию задач управления составом организации: формирование, оптимизация, расширение, сокращение и замена персонала. Важным при управлении составом представляется и вопрос управления качеством персонала организации, т.е. уровнем знаний, умений и навыков, мотивацией сотрудников, их вовлеченностью в деятельность и реализацию целей компании, составом ценностей персонала. Перечень качеств работников, представляющих значимость для эффективной деятельности организации, растет. Сегодня большое внимание уделяется развитию у сотрудников эмпатии, способности к коллаборации. Расширяется содержание понятия интеллекта человека. Теперь оно включает в себя не только уровень IQ индивида, но и дополнилось составляющей эмоционального интеллекта. Все это привело к формированию нового подхода к оценке и управлению персоналом организации, который реализуется на основе понятия человеческого капитала.

Несмотря на достаточно глубокую проработанность вопроса управления составом организационных систем, в свете проблематики адаптивности представляется уместным рассматривать задачу адаптивного управления составом системы. Согласно определению адаптивности, в общем виде адаптивное управление составом организационной системы должно представлять собой управление ее составом при изменяющихся условиях среды (под воздействием помех и возмущений), нацеленное на оптимизацию функционирования и сохранение организации.

Таким образом, модель адаптивного управления составом является частной моделью адаптивности организационной системы.

Модель структуры системы. Части и элементы любой системы не существуют обособленно друг от друга, не находятся в хаотическом состоянии. Системные свойства возникают у некоторого множества элементов, в том числе благодаря упорядоченности отношений между ними, наличию необходимого набора связей (количественного и качественного). Связи элементов системы образуют ее структуру. Понятие структуры системы полноценно изложено в работе [1]: «...структура (от лат. structuraстроение, расположение, порядок) - совокупность устойчивых связей объекта, обеспечивающих его целостность и тождественность самому себе, т.е. сохранение основных свойств при различных внешних и внутренних изменениях». Существуют различные виды структур, поэтому их принято делить на классы. Наиболее известными являются сетевые и иерархические структуры. С помощью сетевых структур удобно представлять декомпозицию объекта во времени. Иерархические структуры используются для отображения пространственных отношений элементов системы.

Ранее уже говорилось, что модель - это копия объекта, включающая только существенные для целей исследования или практики характеристики оригинала. Естественно, этому правилу следуют и при создании моделей структуры системы. Учитывая тот факт, что количество связей между элементами реальных объектов может быть достаточно велико, в их модели включают только 
те отношения, которые необходимы в рамках изучаемого вопроса (или для формирования определенных свойств модели).

Модели структуры систем чаще представляют в графическом виде. Примеры моделей объектов, имеющих различные структуры, приведены на рис. 3.

Учитывая разнообразие отношений, которые возникают в организационных системах между элементами (например, сотрудниками) и подсистемами (например, подразделениями), а также их существенное влияние на результаты деятельности организаций, в целях управления документально закрепляется ряд внутренних структур, в их числе:

- организационная структура (отражает отношения подчиненности подразделений и управления ими);

- функциональная структура (отражает распределение функциональных обязанностей подразделений или персонала);

- штатная структура (отражает состав подразделений, перечень должностей, фонд оплаты труда и размеры должностных окладов).

Создание моделей структуры системы позволяет рассматривать вопрос управления структурой организации. Как указывает Д.А. Новиков в работе [7], в общем случае задача управления структурой организационной системы «...формулируется как зада- ча поиска структуры или набора структур, которая минимизировала бы организационные издержки (или максимизировала некоторый функционал...) при ограничении удовлетворения системой внешним требованиям». Представляется важным добавить к сформулированной задаче управления структурой организационной системы еще одно ограничение, также предлагаемое в работе [7]. Задача управления структурой организационной системы предполагает «...построение рациональной организационной структуры, то есть организационной структуры, наиболее полно соответствующей предназначению организации». Задачи управления структурой пока еще являются малоизученными. Сложность их решения обусловлена значительным количеством факторов, оказывающих влияние на вид оптимальной структуры организации. К их числу относятся, в первую очередь специфика технологического процесса, размер организации, внешние и внутренние ограничения. Следует отметить также высокую степень зависимости оптимального решения задачи управления структурой от решений других задач управления организацией (например, влияние возможностей управления составом организационной системы: формирование, оптимизация, расширение, сокращение и замена персонала).

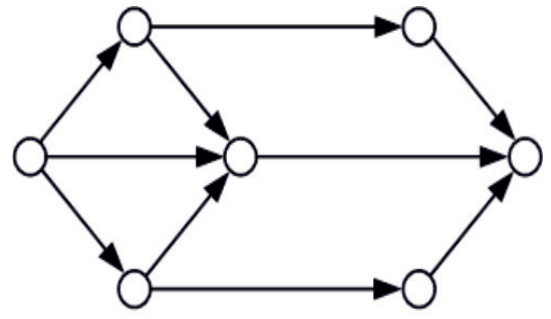

a)

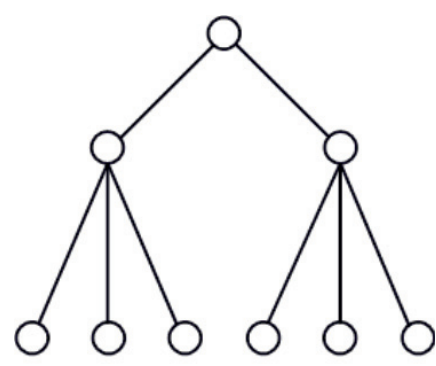

в)

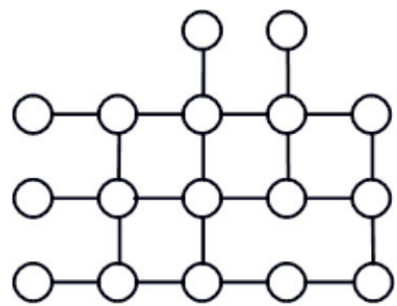

б)

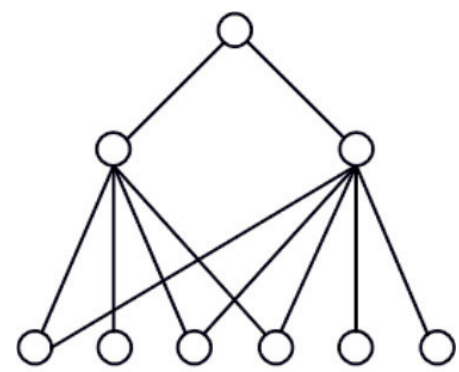

2)

Рис. 3. Модели структуры системь: а) сетевая структура («сеть»); б) матричная структура («матрииа»); в) структура типа «дерево» (иерархическая структура с «сильными» связями); г) иерархическая структура со «слабыми» связями 
Как и в случае адаптивного управления составом, представляется целесообразным рассмотреть задачу адаптивного управления структурой организации. Дополнительным (к накладываемым при управлении составом) ограничением, которое возникает при решении задачи адаптивного управления структурой, является необходимость оптимального управления структурой организационной системы в условиях изменяющихся факторов среды. В отношении модели адаптивного управления структурой можно говорить о том, что она является частной моделью адаптивности организационной системы.

Таким образом, в качестве моделей адаптивности организационной системы на базовых уровнях ее организации могут выступать модели адаптивного управления составом и структурой системы.

\section{Моделирование адаптивных организационных систем}

В предыдущем разделе были рассмотрены модели адаптивности организационных систем на уровне базовых моделей. При этом отмечалось, что количество частных моделей, фиксирующих тот или иной уровень организации системы, обеспечивающий проявление некоторого ее свойства, может быть неограниченным. Действительно, если говорить о сложных системах, к которым относятся и организационные системы, то их способность к реакциям, адекватным окружающей среде, обеспечивается за счет широкого набора разнообразных функций и свойств системы. Это и определяет во3можность создания неограниченного количества частных моделей сложных систем.

Следовательно, для построения адекватной модели адаптивности организационной системы необходимо формирование, по возможности, полного метакортежа, состоящего из частных кортежей частных моделей организационной системы, фиксирующих различные уровни ее организации. Это позволит создать соответствующий метакортеж частных кортежей частных моделей адаптивного управления ключевыми свойствами организационной системы на каждом из ее уровней. Тогда в качестве основной проблемы формирования метакортежа следует рассматривать вопрос выбора уровней, обеспечивающих существенные свойства организационной системы, порядка их иерархии. Безусловно, и состав уровней, и их ранжирование определяются целями решаемой задачи. Кроме того, выбор зависит и от предпочтений исследователя. В качестве примеров различия взглядов на представление внутренней организации сложных систем можно привести наиболее известные:

- теория иерархических многоуровневых систем М. Месаровича;

- система классификации сложных систем К. Боулдинга;

- модель организационной структуры К. Минцберга.

Для нахождения компромисса между простотой описания системы и необходимостью учета многочисленных характеристик сложной системы М. Месарович в работе [8] предлагает использование иерархически упорядоченного семейства моделей: «...система задается семейством моделей, каждая из которых описывает поведение системы с точки зрения различных уровней абстрагирования». В зависимости от отражаемых сущностных особенностей сложных систем в качестве уровней абстрагирования М. Месарович предлагает использовать понятия страт, слоев, эшелонов.

Интересным для решения задачи построения модели адаптивной организационной системы представляется использование системы классификации К. Боулдинга, описанной в работе [9]. В целях изучения системы К. Боулдинг выделил в ней уровни, положив в основу классификации сложность их организации. Каждый уровень отличается степенью его сложности, привносящей новые системные свойства, неразличимые на других уровнях. Система классификации состоит из следующих уровней:

- простая структура (элементный состав и межэлементные связи);

- простой механизм (функционирование);

- динамическая система замкнутая, открытая (изменение во времени, взаимодействие со средой);

- управляемая система (целенаправленность);

- кибернетическая система (множественность целей, самоуправление);

- живая система (гомеостазис, самоорганизация, эволюция);

- организм (взаимодействие самоуправляемых подсистем);

- животное (подсознание, поведение);

- человек (сознание);

- организация (коллективный труд);

- социум (создание искусственных систем - социальных институтов, науки, культуры, религии и т.п.).

Классификационные уровни системы К. Боулдинга могут использоваться для формирования частных кортежей частных моделей организационной системы, на основе которых можно создавать частные кортежи частных моделей адаптивного управления для каждого уровня представления 
организационной системы. Как видим, уже рассмотренные базовые модели организационной системы (состава и структуры системы) соответствуют первому уровню классификации К. Боулдинга.

Применяя систему классификации К. Боулдинга, модель адаптивности организационной системы будет иметь вид метакортежа, включающего частные кортежи, состоящие из следующих частных моделей адаптивного управления на каждом из уровней организационной системы:

- адаптивного управления составом;

- адаптивного управления структурой;

- адаптивного управления функционалом;

- адаптивного управления целями;

- адаптивного управления равновесными состояниями (гомеостазис);

- самоорганизующегося адаптивного управления;

- адаптивного управления развитием (эволюцией);

- адаптивного поведения;

- адаптивного управления коллективным сознанием (интеллектом);

- адаптивного управления совместной деятельностью;

- адаптивного управления социальной структурой (создание организационных институтов, организационной культуры).

Таким образом, для построения модели адаптивной организационной системы могут использоваться различные системы организационной классификации, например предложенные М. Месаровичем, К. Боулдингом и К. Минцбергом. Использование иных систем классификации организаций только дополнит и обогатит модель адаптивной организационной системы. Учитывая, что метакортеж организационной системы является неограниченным множеством частных кортежей частных моделей, представленная модель адаптивности организационной системы не является завершенной.

\section{Ключевая особенность моделей адаптивного управления}

Как отмечалось в работах $[4,10]$, большие и сложные системы (к которым относятся и организационные) имеют огромное число контрагентов и, соответственно, могут быть подвержены влиянию как минимум такого же числа изменений этих факторов окружающей среды. Даже объединение факторов влияния в однородные группы, т.е. классификация, не решает проблему их многочисленности. Но все же не только большое число контрагентов и изменения в их влиянии представляют сложность для управления организационными системами.
Более важным условием для реагирования системы на влияние факторов среды выступает осведомленность о будущих изменениях в их поведении. Как следует из теории автоматического управления, если органу управления известен закон изменения состояний объекта, то управление его поведением может быть реализовано с использованием более простых моделей управления, в том числе без обратной связи (разомкнутый контур). Отсутствие осведомленности о поведении фактора влияния в будущем становится источником неопределенности. В свою очередь, сложность получения информации о закономерностях поведения объекта определяется его природой, а именно - относится ли он к разряду детерминированных или же вероятностных (стохастических) систем. Однако чтобы быть более точным в описании неопределенности, следует сказать, что принято рассматривать несколько ее видов. В работе [4] авторы укрупненно выделяют два больших класса неопределенности: внешнюю и внутреннюю (по отношению к объекту, который испытывает неопределенность). В свою очередь, исходя из природы объекта (т.е. видов законов, описывающих его поведение), внешняя неопределенность бывает интервальной, вероятностной и нечеткой. Кроме того, существуют и иные источники внешней неопределенности. Причинами такой неопределенности могут быть неточности, потери или искажения информации, используемой организационной системой. Появление этих видов внешней неопределенности связано с ограниченными возможностями измерительных приборов, точностью их настройки, наведением помех в каналах передачи информации, возникновением погрешности вычислений, действием внешних агрессивных по отношению к организационной системе факторов и др. К сказанному следует добавить, что внешняя неопределенность проникает в организационную систему по различным каналам. Авторы работы [4] указывают пять таких каналов.

Возникновение внутренней неопределенности связано с проявлением активности элементов организационной системы, вызванной реализацией их индивидуальных интересов. Внутренняя неопределенность с позиции теории игр проявляется в так называемой игре агентов и поэтому получила название игровой.

Еще одним видом неопределенности выступает априорная неопределенность, которая возникает из-за неполноты априорной информации об организационной системе, что не позволяет качественно построить 
модель системы и, соответственно, прогнозировать ее состояние в будущем. Более полно виды неопределенности и каналы их проникновения в организационную систему рассмотрены в работе [4].

Следует отметить, что наличие неопределенности, независимо от ее вида и канала проникновения, представляет основную сложность для обеспечения эффективного управлении системами. Поэтому ее разрешение является ключевой задачей адаптивных организационных систем. С учетом рассматриваемого подхода к моделированию адаптивной организационной системы, предполагающего создание метакортежа частных моделей адаптивного управления, и действия неопределенности различных видов, ключевой особенностью модели адаптивной организации будет являться наличие механизма разрешения неопределенности в каждой из частных моделей адаптивного управления.

Таким образом, неопределенность является одной из основных проблем управления организационными системами, что требует учета при построении моделей адаптивного управления ими. В зависимости от видов и каналов проникновения неопределенности в организационную систему модель адаптивного управления ею должна включать механизмы разрешения соответствующей неопределенности.

\section{Заключение}

1. В качестве одного из положений общего подхода при построении адаптивной организационной системы может рассматриваться формирование метакортежа, состоящего из частных кортежей частных моделей управления адаптивностью на каждом уровне такой системы.

2. Адаптивность организационной системы может быть обеспечена благодаря управлению изменениями системы, что предполагает построение частных моделей адаптивного управления на каждом из уровней системы.

3. Одними из базовых моделей адаптивной организационной системы являются модели адаптивного управления составом и структурой системы.

4. Для построения метакортежа частных кортежей частных моделей адаптивной организационной системы могут использоваться различные системы организационной классификации, в том числе предложенные М. Месаровичем, К. Боулдингом и К. Минцбергом. Метакортеж организационной системы является неограниченным множеством частных кортежей и частных моделей, поэтому модель адаптивности организационной системы предполагает дальнейшее развитие.

5. В связи с тем, что неопределенность является одной из ключевых проблем управления организационными системами, модель адаптивной организационной системы предполагает наличие механизма разрешения неопределенности в каждой из моделей адаптивного управления.

\section{Список литературы}

1. Кориков А.М., Павлов С.Н. Теория систем и системный анализ. - М.: ИНФРА-М, 2014. - 286 с.

2. Бурков В.Н., Коргин Н.А., Новиков Д.А. Введение в теорию управления организационными системами / В.Н. Бурков, Н.А. Коргин, Д.А. Новиков // Под ред. Д.А. Новикова. - М.: Либроком, 2009. - 264 с.

3. Антончиков С.Н. Адаптивность и связи системы// Экономика и управление: проблемы, решения. - 2016. № 12 , т. 2 (60). - С. 11-21.

4. Черников Б.В., Антончиков С.Н. Источники неопределенности в организационных системах и адаптивное управление // Экономика и управление: проблемы, решения. - 2017. - № 4, т. 1 (64). - С. 44-56.

5. Ремонтов А.П., Кузнецова М.В., Хислямова С.В. Теория систем и системный анализ. - Пенза: ПензГТУ, 2015. - 200 с.

6. Туликов А.Н. Теория систем и системный анализ. Абакан: ХТИ - фил. СФУ, 2013. - 143 с.

7. Новиков Д.А. Теория управления организационными системами: учеб.-метод. пособие. - М.: Моск. психол.-соц. ин-т, 2005. - 584 с.

8. Месарович М., Мако Д., Такахара И. Теория иерархических многоуровневых систем/ Пер. с англ. под ред. И.Ф. Шахнова. - М.: Мир, 1973. - 344 с.

9. Воронин А.А., Губко М.В., Мишин С.П., Новиков Д.А. Математические модели организаций: учебное пособие. - М.: ЛЕНАНД, 2008. - 360 с.

10. Черников Б.В., Антончиков С.Н. Разрешение неопределенности - ключевая задача адаптивных систем // Фундаментальные исследования. 2016. - № 12-5. - С. 1167-1178. 